\title{
SOME COMPUTATIONAL ASPECTS OF VEHICLE SHELL FRAMES OPTIMIZATION SUBJECTED TO FATIGUE LIFE
}

The paper deals with the implementation of the fatigue damage conditions into structural optimization process. The study considers a shell finite element structural analysis in conjunction with the multiaxial rainflow counting, the fatigue damage prediction and naturally the sizing (element thickness) optimizing design. We will analyze FE models under random excitation in time domain. The presented optimizing approach will be implemented into solution program compiled in Matlab.

Keywords: finite element analysis, optimization algorithm, multiaxial rainflow counting, fatigue damage

\section{Introduction}

Nowadays it is almost impossible to pick up a journal or conference focused on computational mechanics that doesn't contain some reference to structural optimizing. Although it is possible to design machine parameters by experience it is much better and more effective to predict the basic properties of the new designed structure by using optimizing procedure which is generally based on a series of controlled computing analyses [1].

\section{Chosen methods for multiaxial fatigue damage prediction}

To calculate the structural mass (or volume) is not a complicated problem but the constrain conditions usually depend on FE analysis, identification of a "damage" critical points and multiaxial fatigue prediction [12]. Let's now focus on the cumulative damage counting by using multiaxial rainflow decomposition of the stress response. It should be noted $[4,12]$ that the fatigue damage calculation of the machine parts is generally problematic because the results are considerable changed in the principal stresses $[2,5]$. Using FE analysis we can get six components of the stress-time function (multiaxial stress) but it is very difficult to obtain an equivalent - uniaxial load spectrum by reason of comparison with applied computational fatigue curve. In our case the rainflow analysis for random stresses known in classic uniaxial form as von Mises or Tresca hypotheses is impossible. It means that the important goal of this part will be to propose some approaches to estimate the high-cycle fatigue damage for multiaxial stresses caused by random vibration analysed structure $[7,11]$. Generally we can apply two fundamental approaches for multiaxial rainflow counting:

- Critical Plane Approach (CPA) [11] and

- Integral Approach (IA) [7].
It is well-known that the Wöhler curve (Fig. 1, sometimes called S-2N curve) is basic source of getting information of the material fatigue life. Generally the S-2N curve is statistically evaluated by experimental fatigue curve. This is a graph of the magnitude of a cyclical nominal stress $\sigma_{A}$ against the logarithmic scale of cycles to failure $2 N_{f}$. It is advantage to show it in logarithmical or semi logarithmical coordinates.

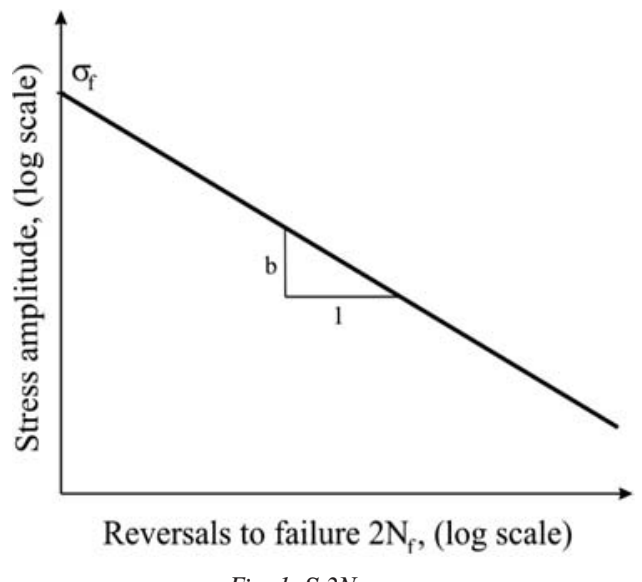

Fig. 1. S-2N curve

The $\sigma_{A}-2 N_{f}$ relation can be written as follows

$\sigma_{A}=\sigma_{f} \cdot\left(2 N_{f}\right)^{b}$

where $\sigma_{f}$ is the fatigue stress coefficient, $2 N_{f}$ is number of cycles to failure, $b$ is fatigue strength exponent and $\sigma_{A}$ is stress amplitude

\footnotetext{
* Milan Saga, Peter Kopas, Milan Vasko

Department of Applied Mechanics, Faculty of Mechanical Engineering, University of Zilina, Slovakia, E-mail: milan.saga@fstroj.uniza.sk
} 
to failure. Some researches rewrite the relationship (1) into following form

$$
\sigma_{A}^{m} \cdot\left(2 N_{f}\right)=K
$$

where $m=-(1 / b)$ and $K=\sigma_{f}^{(-1 / b)}=\sigma_{f}^{m}$

Considering the mean stress modified version of the stress amplitude (using Goodman, Soderberg, Geber), eq. 2 can be rewritten as follows

$$
\left\{\sigma_{A} \cdot\left[1-\left(\frac{\sigma_{M}}{R_{F}}\right)^{k}\right]^{-1}\right\}^{m} \cdot\left(2 N_{f}\right)=K .
$$

If $k=1$ and $R_{F}=R_{E}$ (yield stress) the Soderberg's model is used, if $k=1$ and $R_{F}=R_{M}$ (strength limit) the Goodman's model is used and if $k=2$ a $R_{F}=R_{M}$ the Geber's model is used. Using the linear Palmgren-Miner law we can calculate fatigue damage for stress amplitude $\sigma \mathrm{Ai}$ as follows

$$
d_{i}=\frac{1}{2 N_{f-i}}=\left\{\frac{\sigma_{A i}}{\sigma_{f}} \cdot\left[1-\left(\frac{\sigma_{M}}{R_{F}}\right)^{k}\right]^{-1}\right\}^{m} .
$$

\section{Damage calculation for thin shell finite element using CPA}

Let us consider well-known shell finite elements (Kirchhoff's or Mindlin's formulation) [1, 9, 11]. The stiffness parameters depend on material constants and element geometry, mainly on its thickness. At first we have to prepare the stress calculation process. This process is based on the expression of the $j$-th element membrane forces and bending moments (without shear forces) [10,11] i.e.

$$
\begin{aligned}
& {\left[\begin{array}{lll}
F_{x x} & F_{y y} & F_{x y}
\end{array}\right]_{j}^{T}=\boldsymbol{F}_{m}^{j}=\int_{S} \boldsymbol{E}_{m}^{j} \cdot \varepsilon_{m}^{j} d S_{j}=} \\
& =\boldsymbol{E}_{m}^{j} \cdot \int_{S} \boldsymbol{B}_{m}^{j} d S_{j} \cdot \boldsymbol{u}_{L}^{j}=t_{j} \cdot \boldsymbol{D}_{j} \cdot \boldsymbol{I}_{m}^{j} \cdot \boldsymbol{u}_{L}^{j}
\end{aligned}
$$

and

$$
\begin{aligned}
& {\left[\begin{array}{lll}
M_{x x} & M_{y y} & M_{x y}
\end{array}\right]_{j}^{T}=\boldsymbol{M}_{b}^{j}=\int_{S} \boldsymbol{E}_{b}^{j} \cdot \varepsilon_{b}^{j} d S_{j}=} \\
& =\boldsymbol{E}_{b}^{j} \cdot \int_{S} \boldsymbol{B}_{b}^{j} d S_{j} \cdot \boldsymbol{u}_{L}^{j}=\frac{t_{j}^{3}}{12} \cdot \boldsymbol{D}_{j} \cdot \boldsymbol{I}_{b}^{j} \cdot \boldsymbol{u}_{L}^{j}
\end{aligned}
$$

The auxiliary matrices Im and Ib can be calculated only using the numerical approach. Further details about Em, Eb, D, Bm, $\mathrm{Bb}$, uel and $\mathrm{t}$ are presented in [10]. The extreme stress values can be expected at the top or at the bottom surface. Generally, it means or

$$
\sigma_{m b_{-} L}^{j}=\boldsymbol{C}_{L}^{j} \cdot \boldsymbol{f}_{L}^{j}
$$

Let's build new material and auxiliary matrices

$$
\boldsymbol{E}_{m b}=\left[\begin{array}{cc}
t_{j} \cdot \boldsymbol{I}_{3} & \boldsymbol{0}_{3} \\
\boldsymbol{0}_{3} & \frac{\boldsymbol{t}_{j}^{3}}{12} \cdot \boldsymbol{I}_{3}
\end{array}\right] \cdot\left\{\begin{array}{l}
\boldsymbol{D} \\
\boldsymbol{D}
\end{array}\right\}_{j}=\boldsymbol{D}_{\boldsymbol{t}} \cdot \boldsymbol{D}_{m b}, \quad \boldsymbol{I}_{m b}=\left\{\begin{array}{l}
\boldsymbol{I}_{m}^{j} \\
\boldsymbol{I}_{b}^{j}
\end{array}\right\},
$$

where the matrix $I_{3}$ is the classical unit matrix. Then (7) can be rewritten as follows

$$
\begin{aligned}
& \left.\sigma_{j \_m b}\right|_{t o p}=\boldsymbol{A}_{t, t o p} \cdot \boldsymbol{E}_{m b} \cdot \boldsymbol{I}_{m b} \cdot \boldsymbol{u}_{L}^{J}=\boldsymbol{A}_{t, t o p} \cdot \boldsymbol{D}_{t} \cdot \boldsymbol{D}_{m b} \cdot \boldsymbol{I}_{m b} \cdot \boldsymbol{u}_{L}^{J},(10 \mathrm{a}) \\
& \left.\sigma_{j \_m b}\right|_{b o t}=\boldsymbol{A}_{t, b o t} \cdot \boldsymbol{E}_{m b} \cdot \boldsymbol{I}_{m b} \cdot \boldsymbol{u}_{L}^{J}=\boldsymbol{A}_{t, b o t} \cdot \boldsymbol{D}_{t} \cdot \boldsymbol{D}_{m b} \cdot \boldsymbol{I}_{m b} \cdot \boldsymbol{u}_{L}^{J} \cdot(10 \mathrm{~b})
\end{aligned}
$$

\section{Findley hypothesis}

Findley has assumed the critical plane as a plane with maximum shear stress, i.e. the fatigue equivalent shear stress can be written as follows [11]

$$
\tau_{A_{-} F i n}=\tau_{\max }+k \cdot \sigma_{m}
$$

where $k$ is Findley's factor whose value for tough metal can be about 0.3. Using von Mises relationship between normal and shear stresses and classical plane stress analysis for top or bottom element surface it is possible to rewrite (9) into the following form

$$
\begin{aligned}
& \left.\sigma_{A_{-} \text {Fin }}^{j}\right|_{\text {top }}=\sqrt{3} \cdot\left\{\operatorname { s i g n } ( T _ { 2 } \cdot \sigma _ { j _ { - } m b } | _ { t o p } ) \cdot \left[\left(\left.\sigma_{j_{-} m b}\right|_{t o p}\right)^{T} \cdot T_{1} \cdot\right.\right. \\
& \left.\left.\cdot\left(\left.\sigma_{j \_m b}\right|_{t o p}\right)\right]^{-1 / 2}+\left.k \cdot T_{2} \cdot \sigma_{j \_m b}\right|_{\text {top }}\right\} \text {, } \\
& \left.\sigma_{A_{-} F i n}^{j}\right|_{b o t}=\sqrt{3} \cdot\left\{\operatorname { s i g n } ( T _ { 2 } \cdot \sigma _ { j _ { - } m b } | b o t ) \cdot \left[\left(\sigma_{j_{-} m b} \mid b o t\right)^{T} \cdot T_{1}\right.\right. \\
& \left.\left.\cdot\left(\sigma_{j \_m b} \mid b o t\right)\right]^{-1 / 2}+k \cdot T_{2} \cdot \sigma_{j \_m b} \mid b_{b o t}\right\} \text {, }
\end{aligned}
$$

where

$$
T_{1}=\left[\begin{array}{ccc}
0,25 & -0,25 & 0 \\
-0,25 & 0,25 & 0 \\
0 & 0 & 1
\end{array}\right] \text { and } T_{2}=\left[\begin{array}{lll}
0,5 & 0,5 & 0
\end{array}\right]
$$

The damage calculation can be realised using eq. (4). The presented relationships were applicable for FE analyses. Numerical and experimental tests confirmed that the factor $k=0.3$ was overstated $[4,11]$ by the author.

$$
\left[\begin{array}{l}
\sigma_{m b} \mid t o p \\
\sigma_{m b} \mid b o t
\end{array}\right]^{j}=\left\{\begin{array}{l}
\sigma_{x x, t o p} \\
\sigma_{y y, t o p} \\
\sigma_{x y, t o p} \\
\sigma_{x x, b o t} \\
\sigma_{y y, b o t} \\
\sigma_{x y, b o t}
\end{array}\right\}^{j}=\left[\begin{array}{cccccc}
1 / t_{j} & 0 & 0 & 6 / t_{j}^{2} & 0 & 0 \\
0 & 1 / t_{j} & 0 & 0 & 6 / t_{j}^{2} & 0 \\
0 & 0 & 1 / t_{j} & 0 & 0 & 6 / t_{j}^{2} \\
1 / t_{j} & 0 & 0 & -6 / t_{j}^{2} & 0 & 0 \\
0 & 1 / t_{j} & 0 & 0 & -6 / t_{j}^{2} & 0 \\
0 & 0 & 1 / t_{j} & 0 & 0 & -6 / t_{j}^{2}
\end{array}\right] \cdot\left\{\begin{array}{l}
F_{x x} \\
F_{y y} \\
F_{x y} \\
M_{x x} \\
M_{y y} \\
M_{x y}
\end{array}\right\}=\left[\begin{array}{l}
A_{t, t o p} \\
A_{t, b o t}
\end{array}\right]^{j} \cdot\left\{\begin{array}{l}
F_{m} \\
M_{b}
\end{array}\right\}^{j},
$$




\section{Dang Van hypothesis}

Dang Van again assumed the critical plane with shear stress but with difference in factor $k$, which can be calculated from normal and shear fatigue limit, i.e.

$$
\tau_{A_{-} D V}=\tau_{\max }+k \cdot p_{m}=\frac{\sigma_{1}-\sigma_{3}}{2}+\frac{\tau_{C}-\frac{\sigma_{C}}{2}}{\frac{\sigma_{C}}{\sqrt{3}}} .
$$

$$
\frac{\sigma_{1}+\sigma_{2}+\sigma_{3}}{3},
$$

where $\tau_{C}$ is shear (torsional) fatigue limit, $\sigma_{C}$ is normal (axial) fatigue limit, $\sigma_{1}, \sigma_{2}, \sigma_{3}$ are principal stresses. Relationship (11) is possible to use like that

$$
\begin{aligned}
& \sigma_{A_{-} D V}=\sqrt{3} \cdot\left[\operatorname{sign}\left(\frac{\sigma_{1}-\sigma_{3}}{2}\right) \cdot \frac{\sigma_{1}-\sigma_{3}}{2}+\frac{\tau_{C}-\frac{\sigma_{C}}{2}}{\frac{\sigma_{C}}{\sqrt{3}}} .\right. \\
& \left.\frac{\sigma_{1}+\sigma_{2}+\sigma_{3}}{3}\right] .
\end{aligned}
$$

Using von Mises hypothesis we can get [4]

$$
\frac{\tau_{c}-\frac{\sigma_{C}}{2}}{\frac{\sigma_{C}}{\sqrt{3}}} \approx \frac{\tau_{c}-\frac{\sqrt{3} \tau_{c}}{2}}{\frac{\sqrt{3} \tau_{c}}{3}} \approx 0,232 .
$$

Using the application of the shell stress theory and eqs. 10a, b we can obtain

$$
\begin{aligned}
& \left.\sigma_{A_{-} D V}^{j}\right|_{t o p}=\sqrt{3} \cdot\left\{\operatorname { s i g n } ( T _ { 3 } \cdot \sigma _ { j _ { - } m b } | _ { t o p } ) \left[\left(\left.\sigma_{j_{-} m b}\right|_{t o p}\right)^{T} \cdot T_{1} \cdot\right.\right. \\
& \left.\left.\cdot\left(\left.\sigma_{j_{-} m b}\right|_{t o p}\right)\right]^{-1 / 2}+0,\left.232 \cdot T_{3} \cdot \sigma_{j_{-} m b}\right|_{t o p}\right\}, \\
& \left.\sigma_{A_{-} D V}^{j}\right|_{b o t}=\sqrt{3} \cdot\left\{\operatorname { s i g n } ( T _ { 3 } \cdot \sigma _ { j _ { - } m b } | _ { b o t } ) \cdot \left[\left(\left.\sigma_{j_{-} m b}\right|_{b o t}\right)^{T} \cdot T_{1} \cdot\right.\right. \\
& \left.\left.\cdot\left(\left.\sigma_{j_{-} m b}\right|_{b o t}\right)\right]^{-1 / 2}+0,\left.232 \cdot T_{3} \cdot \sigma_{j_{-} m b}\right|_{b o t}\right\} .
\end{aligned}
$$

Relations (12) and (17) present equivalent stresses applicable for rainflow decomposition for both proportional and non-proportional loading. The cumulative damage calculation can be realised using eq. (4) again.

\section{HMH modified hypothesis}

Applying von Mises equivalent stress for CPA we can obtain the following relationship

$$
\sigma_{A_{-} H M H}=\operatorname{sign}\left[\sigma\left(\boldsymbol{n}_{C P A}\right)\right] \cdot \sqrt{\sigma\left(\boldsymbol{n}_{C P A}\right)^{2}+3 \cdot \tau\left(\boldsymbol{n}_{C P A}\right)^{2}},
$$

or in detail

$$
\begin{aligned}
& \left.\sigma_{A_{-} H M H}^{j}\right|_{t o p}\left(\boldsymbol{n}_{C P A}\right)=\operatorname{sign}\left[\left.\left.\sigma_{j \_m b}^{T}\right|_{t o p}\left(\boldsymbol{n}_{C P A}\right) \cdot T_{\sigma} \cdot \sigma_{j \_m b}^{T}\right|_{t o p}\left(\boldsymbol{n}_{C P A}\right)\right] . \\
& \cdot\left[\left.\left.\sigma_{j \_m b}^{T}\right|_{t o p}\left(\boldsymbol{n}_{C P A}\right) \cdot T_{4} \cdot \sigma_{j \_m b}^{T}\right|_{t o p}\left(\boldsymbol{n}_{C P A}\right)\right]^{-1 / 2}
\end{aligned}
$$

$$
\begin{aligned}
& \left.\sigma_{A_{-} H M H}^{j}\right|_{b o t}\left(\boldsymbol{n}_{C P A}\right)=\operatorname{sign}\left[\sigma_{j \_m b}^{T} \mid b o t\right. \\
& \left.\left.\cdot\left[\boldsymbol{n}_{C P A}\right) \cdot T_{\sigma} \cdot \sigma_{j_{-} m b}^{T}\right|_{b o t}\left(\boldsymbol{n}_{C P A}\right)\right] \\
& {\left[\sigma_{j \_m b} \mid b o t\right.} \\
& \left.\boldsymbol{n}_{C P A}\right) \cdot T_{4} \cdot \sigma_{j \_m b}^{T} \mid b o t \\
& \left.\left.\boldsymbol{n}_{C P A}\right)\right]^{-1 / 2}
\end{aligned}
$$

where

$$
T_{4}=\left[\begin{array}{ccc}
1 & -0.5 & 0 \\
-0.5 & 1 & 0 \\
0 & 0 & 3
\end{array}\right] \text { and } T_{\sigma}=\left[\begin{array}{ccc}
\sigma_{x} & \tau_{x y} & 0 \\
\tau_{x y} & \sigma_{y} & 0 \\
0 & 0 & 0
\end{array}\right]
$$

In this case it should be noted that computational approach depends on a searching process of a critical plane normal vector $\boldsymbol{n}_{C P A}$. If the rainflow analysis is used it is very important to know the sign of the calculated equivalent stress therefore the sign of this stress is defined by sign of normal component. For searching process was used optimizing tools in Matlab [12] and optimizing problem for cumulative damage function is usually formulated as follows

$$
\begin{aligned}
& \left.D_{i_{-} \max }\right|_{H M H}=\max \left[\sum_{j=1}^{m c} d_{j}\left(\boldsymbol{n}_{C P A}\right)\right]= \\
& =\max \left\{\left\{\frac{\left.\sigma_{A_{-} H M H}^{i}\right|_{b o t}\left(\boldsymbol{n}_{C P A}\right)_{j}}{\sigma_{f}} \cdot\left[1-\left(\frac{\left.\sigma_{M_{-} H M H}^{i}\right|_{b o t}\left(n_{C{ }_{C A}}\right)_{j}}{R_{f}}\right)^{k}\right]^{-1}\right\}\right.
\end{aligned}
$$

for unknown vector $\boldsymbol{n}_{C P A}$ and stresses on bottom surface. The same computational process can be realised on top element surface.

\section{Damage calculation for thin shell finite element using IA}

The fundamental idea is to count rainflow cycles on all linear combinations of the stress random vector components [7], i.e.

$$
\begin{aligned}
& \sigma_{A_{-} M R F}(t)=c_{1} \cdot \sigma_{x}(t)+c_{2} \cdot \sigma_{y}(t)+c_{3} \cdot \sigma_{z}(t)+ \\
& +c_{4} \cdot \tau_{x y}(t)+c_{5} \cdot \tau_{y z}(t)+c_{6} \cdot \tau_{z x}(t)
\end{aligned}
$$

on the assumption that the parameters ci belong to a hypersphere $\sum_{i=1}^{n} c_{i}^{2}=1$. Practically if the stress state is biaxial (e.g. thin shell finite element) the stress components can be written under the form of three dimension vector $\sigma=\left[\sigma_{x}, \sigma_{y}, \tau_{x y}\right]^{T}$ and the equivalent stress will be calculated as follows

$$
\sigma_{A_{\_} M R F}(t)=c_{1} \cdot \sigma_{x}(t)+c_{2} \cdot \sigma_{y}(t)+c_{3} \cdot \tau_{x y}(t)=c \cdot \sigma
$$

on condition that $c_{1}^{2}+c_{2}^{2}+c_{3}^{2}=1$. In the case of shell element we can obtain again the following relationships

$$
\begin{aligned}
& \left.\sigma_{A_{-} M R F}^{j}\right|_{t o p}(\boldsymbol{c})=\left.\boldsymbol{c} \cdot \boldsymbol{\sigma}_{j_{\perp} m b}\right|_{t o p}(\boldsymbol{c}) \quad \text { and } \\
& \left.\boldsymbol{\sigma}_{A_{-} M R F}^{j}\right|_{b o t}(\boldsymbol{c})=\left.\boldsymbol{c} \cdot \boldsymbol{\sigma}_{j \_m b}\right|_{b o t}(\boldsymbol{c})
\end{aligned}
$$

Hence the next goal will be to find extreme value of the estimated damage for vector $\mathrm{c}$ and $i$-th element, i.e. 


$$
\begin{aligned}
& \left.D_{i_{-} \max }\right|_{M R F}=\max \left[\sum_{j=1}^{m c} d_{j}(\boldsymbol{c})\right]= \\
& =\max \left\{\left\{\frac{\left.\sigma_{A_{-} M R F}^{i}\right|_{b o t}(c)_{j}}{\sigma_{f}} \cdot\left[1-\left(\frac{\left.\sigma_{M_{-} M R F}^{i}\right|_{b o t}(c)_{j}}{R_{f}}\right)^{k}\right]^{-1}\right\}^{m}\right\}
\end{aligned}
$$

where $D_{i-\max } /_{M R F}$ is the maximum value of the cumulative damage for $i$-th element, $2 N_{i}$ is the number of cycles to failure, $m c$ is the number of cycles after rain-flow decomposition of the stress. Naturally we have to observe the normality condition for $\mathrm{c}$ using the following transformation

$$
c=\frac{c^{\prime}}{\sqrt{c^{\prime T} \cdot c^{\prime}}} .
$$

The searching process is realized by computational program FAT_MRFA developed in Matlab. Program calculates elements damage from stress response using original optimizing multiaxial rainflow procedure suggested by authors $[4,6,12]$.

\section{Formulation of the optimizing problem}

Nowadays the optimizing problem of the structural mass minimizing subjected to the prescribed fatigue damage is topical [3]. The optimization problem with optimizing variables $\boldsymbol{x}$ (element thicknesses) can be mathematically stated as follows

$$
F(x)=\sum_{i=1}^{n} \rho_{i} \cdot l_{i} \cdot X_{i} \rightarrow \min ,
$$

subjected to

$$
D_{\text {max }}^{k}(x)-D_{p} \leq 0, \quad k=1 \ldots \mathrm{m}
$$

where $n$ is number of the elements, $m$ is number of the element groups, $D_{P}$ is the prescribed cumulative damage, $D_{\max }^{k}$ is the calculated extreme value of the cumulative damage for $k$-th element group. Another formulation can be based on the idea to built-in damage conditions into objective function, i.e.

$$
F(x)=\sum_{k=1}^{m}\left[1-\frac{D_{k}(x)}{D_{P}}\right] \rightarrow \min .
$$

It is necessary to note that the identification of the extreme element (or node) cumulative damage is generally very time-consuming hence the stress sensitivity or signification analysis plays important role. By reason that the shell structures are in our attention, the signification analysis of the thin shell finite elements will be presented. Theory of this process is in detail published in [11, 12].

\section{Optimization of the track maintenance machine frame}

Let's realise the optimum design of the chosen parameters of the track maintenance machine VKL 400 (Fig. 2) [11]. The computational model creation, the analysis of the vertical and transversal stochastic vibrations of the model and the process of structural designing for a vehicle speed of 40, 70 and $100 \mathrm{kmph}$ will be presented. The cumulative damage determination and design of the cross sections (thicknesses) of the machine frame will be the gist of the solution.

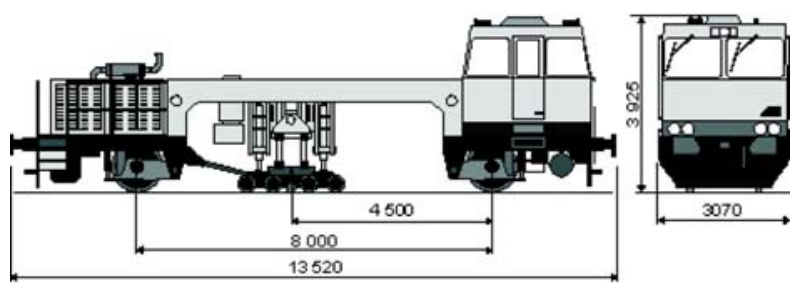

Fig. 2 The basic geometry of the maintenance machine VKL 400

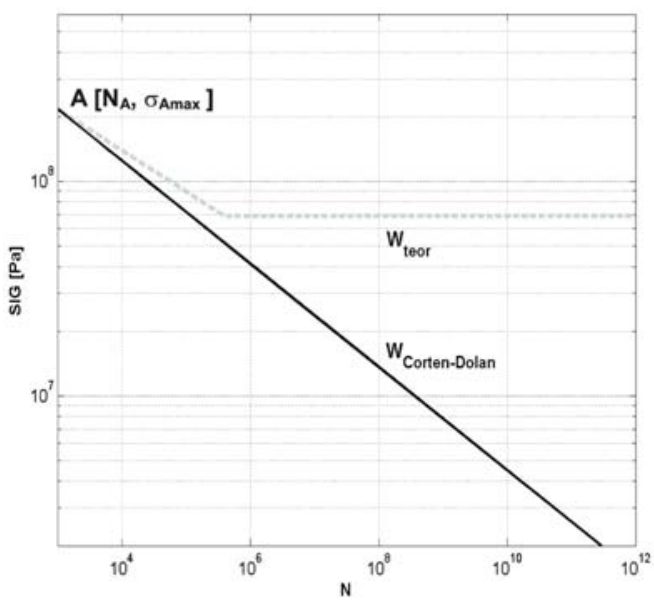

Fig. 3 The "working" $S-N$ curve

\section{Applied material characteristics}

The material computational parameters are Young's modulus $E=2.10^{11} \mathrm{~Pa}$, Poisson ratio $\mu=0.3$, density $\rho=7800 \mathrm{~kg} / \mathrm{m}^{3}$, point of $S-N$ curve $N A=10^{3}$ cycles, $\sigma_{\text {Amax }}=217 \mathrm{MPa}$, Fatigue limit $\sigma_{C}=68.7 \mathrm{MPa}, C-D$ constant $k=0.8$, exponent of $S-N$ curve $m=5.2$. Graphical presentation of the "working" Wöhler curve reduced according to Corten-Dolan is in Fig. 3.

\section{Computational model}

The computational FE model (Fig. 4) was built-up from a virtual model created in PRO/Engineer (Fig. 5) [11]. The selected values describing physical properties of the computing model were parameterized in order to their arbitrary changes. The goal of parameterization was to achieve the maximum variability of the model which related mainly to verification and debugging of this model and consequently to the optimization process. Additional vehicle parameters were considered as follows: the stiffness of vertical primary spring $k_{1}=360000 \mathrm{~N} / \mathrm{m}$, the damping coefficient for vertical primary spring $b_{1}=16000 \mathrm{~N} . \mathrm{s} / \mathrm{m}$, the stiffness of vertical secondary spring $k_{2}=600000 \mathrm{~N} / \mathrm{m}$, damping coefficient for vertical secondary spring $b_{2}=900 \mathrm{~N} . \mathrm{s} / \mathrm{m}$. 


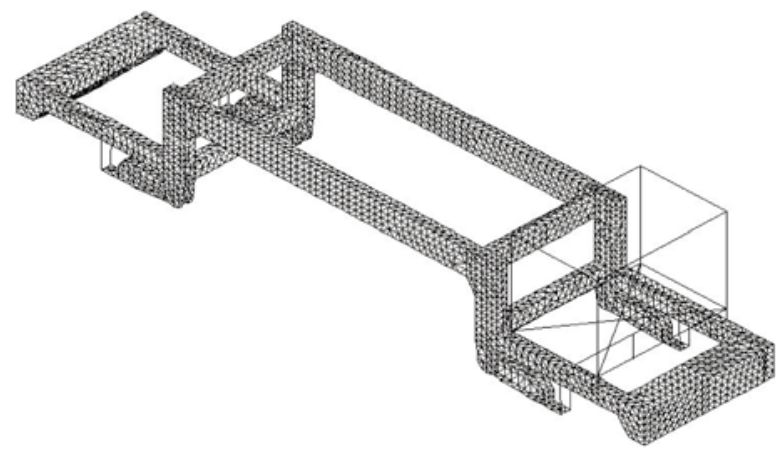

Fig. 4 The finite element model in COSMOS/M

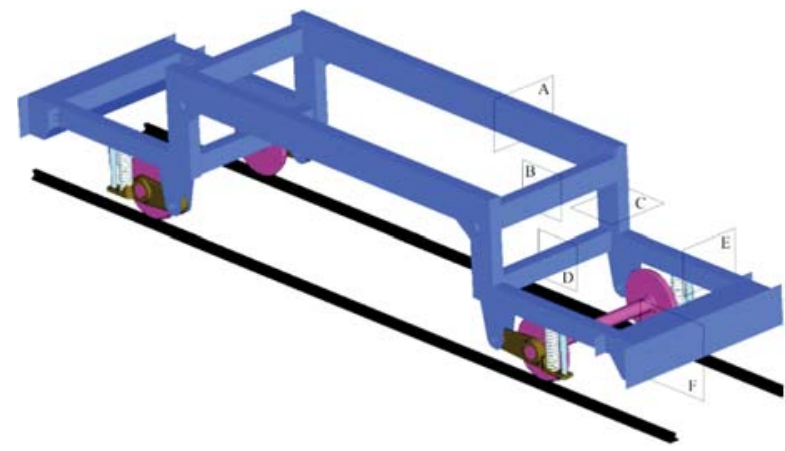

Fig. 5 Model of the analyzed vehicle frame with cross sections identification

\section{Optimizing parameters}

The objective function and constrain conditions were defined by equations (1) and (2). Cross-section parameters, i.e. the thickness of the welded frame plates were been parameterized (Fig. 6). Values used on the build of the frame were applied in the initial analysis, of course. The list of these values is presented in Table 1.

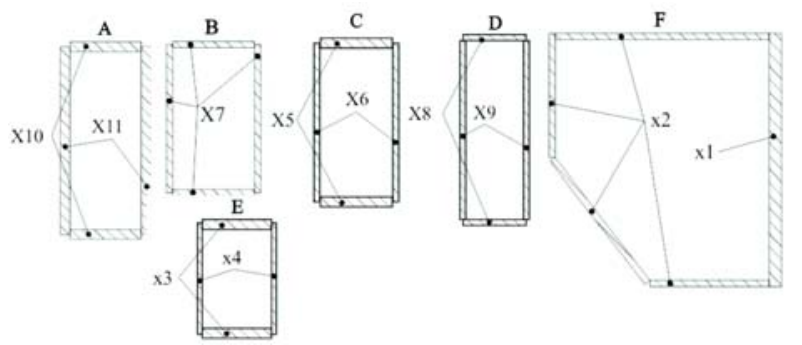

Fig. 6 Variables of the frame cross sections

It should be noted that in each optimizing step the stress response and cumulative damage were calculated for each element group, i.e. for 11 groups. Identification of the damage critical finite
Initial values of design variables

Tab. 1.

\begin{tabular}{|c|c|c|c|c|c|c|c|c|c|c|c|}
\hline Variable & $\mathrm{X} 1$ & $\mathrm{X} 2$ & $\mathrm{X} 3$ & $\mathrm{X} 4$ & $\mathrm{X} 5$ & $\mathrm{X} 6$ & $\mathrm{X} 7$ & $\mathrm{X} 8$ & $\mathrm{X} 9$ & $\mathrm{X} 10$ & $\mathrm{X} 11$ \\
\hline $\begin{array}{c}\text { Value } \\
{[\mathrm{mm}]}\end{array}$ & 30 & 20 & 25 & 20 & 35 & 35 & 15 & 25 & 15 & 25 & 25 \\
\hline
\end{tabular}

elements was realised using classical static analysis $[8,11]$. The results of this process are presented in Table 2.

Numbers of critical elements

Tab. 2.

\begin{tabular}{|c|c|c|c|c|c|c|c|c|c|c|c|}
\hline $\begin{array}{c}\text { Varia- } \\
\text { ble }\end{array}$ & X1 & $\mathrm{X} 2$ & $\mathrm{X} 3$ & $\mathrm{X} 4$ & $\mathrm{X} 5$ & $\mathrm{X} 6$ & $\mathrm{X} 7$ & $\mathrm{X} 8$ & $\mathrm{X} 9$ & $\mathrm{X} 10$ & $\mathrm{X} 11$ \\
\hline $\begin{array}{c}\text { Elem. } \\
\text { No. }\end{array}$ & 74 & 802 & 1753 & 2433 & 3420 & 3639 & 4007 & 4655 & 4939 & 5117 & 7252 \\
\hline
\end{tabular}

The optimization problem was defined as follows

- weight minimization of the frame structures,

- regarding boundary condition - maximum value of the fatigue damage $D p=0.6$,

- 11 optimization parameters - thicknesses (X1 - X11).

Optimization variables can gain the discrete values listed in Table 3.

\section{Model of stochastic excitation}

The stochastic character of excitation was modelled on the basis of the vertical and transversal track unevenness obtained from the measuring on real track $[7,11]$. The behaviour of the chosen random kinematic excitation function is shown in Fig. 8. The points where these functions are input into the computational model are presented in Fig. 7.

Where:

$v \quad$ - vehicle speed,

$L \quad$ - wheel base $(8 \mathrm{~m})$,

$u_{y L}{ }^{(1)}$ - unevenness of the left rail in transverse direction for the front axle,

$u_{z L}{ }^{(1)}-$ unevenness of the left rail in vertical direction for the front axle,

$u_{y P}^{(1)}$ - unevenness of the right rail in transverse direction for the front axle,

$u_{z P}{ }^{(1)}-$ unevenness of the right rail in vertical direction for the front axle,

$u_{y L}{ }^{(2)}$ - unevenness of the left rail in transverse direction for the back axle, i.e. $u_{y L}^{(2)}(t)=u_{y L}^{(1)}\left(t-\frac{L}{v}\right)$

$u_{z L}{ }^{(2)}-$ unevenness of the left rail in vertical direction for the back axle, i.e. $u_{z L}^{(2)}(t)=u_{z L}^{(1)}\left(t-\frac{L}{v}\right)$,

$u_{y P}^{(2)}-$ unevenness of the right rail in transverse direction for the back axle, i.e. $u_{y P}^{(2)}(t)=u_{y P}^{(1)}\left(t-\frac{L}{v}\right)$, 
$u_{z P}{ }^{(2)}-$ unevenness of the right rail in vertical direction for the back axle, i.e. $u_{z P}^{(2)}(t)=u_{z P}^{(1)}\left(t-\frac{L}{v}\right)$.

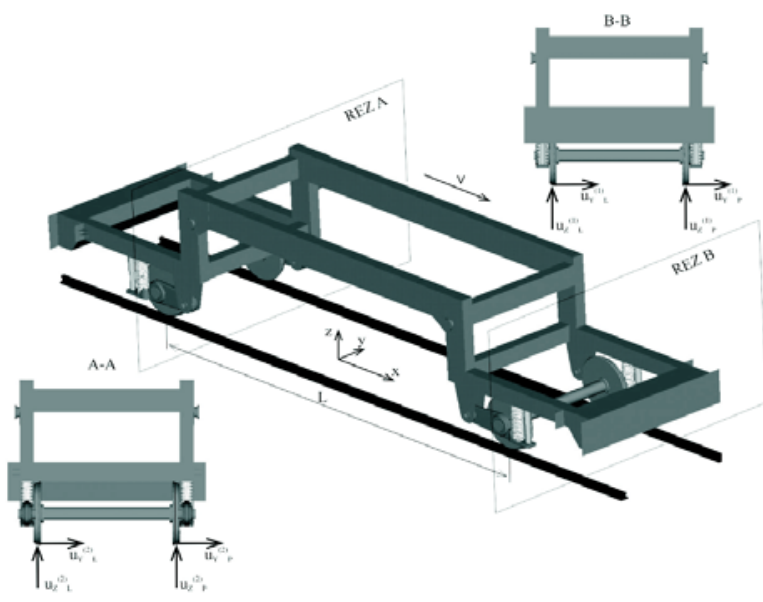

Fig. 7 Identification of the kinematic excitation functions

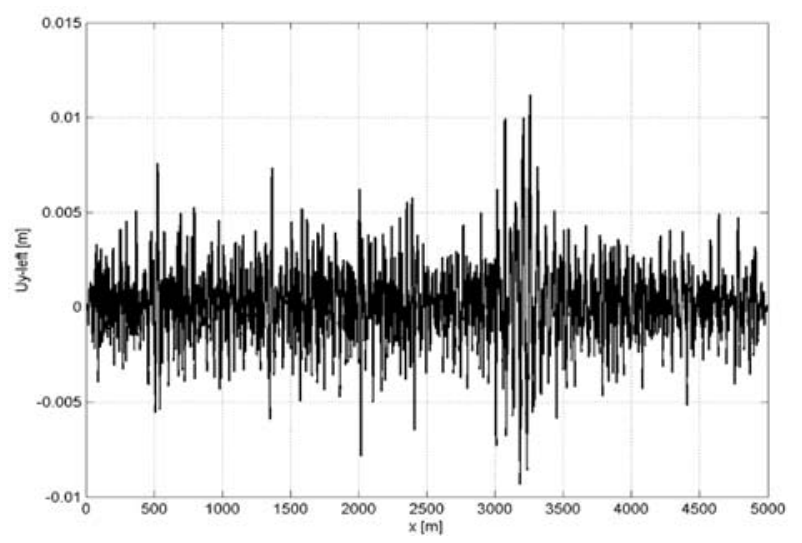

Fig. 8 The random function $-u_{y L}{ }^{(1)}$

Following operating conditions were assumed

- movement 27.000 hours with the velocity of $40 \mathrm{kmph}$,

- movement 18.000 hours with the velocity of $70 \mathrm{kmph}$,

- movement 9.000 hours with the velocity of $100 \mathrm{kmph}$.

\section{Results of the optimizing process}

The optimizing process was very time consuming and contained a lot of computational procedures. The main goal - structural weight reduction was reached. Process of the weight reduction is shown in Fig. 9. The calculation of the cumulative damage for non-proportional shell stresses using IA was one of the most complicated parts of the whole analysis [12]. The convergence history of the optimizing process for chosen optimizing variables and corresponding cumulative damages are presented in Fig. 10. It can be seen that the proposed algorithm is effective in view of a number of design variables $(n v a r=11)$, i.e. the number of iteration steps was very low. Table 3 contains optimum values of the sheet thicknesses.

Optimum values

Tab. 3 .

\begin{tabular}{|c|c|c|c|c|c|c|c|c|c|c|c|}
\hline Variable & $\mathrm{X} 1$ & $\mathrm{X} 2$ & $\mathrm{X} 3$ & $\mathrm{X} 4$ & $\mathrm{X} 5$ & $\mathrm{X} 6$ & $\mathrm{X} 7$ & $\mathrm{X} 8$ & $\mathrm{X} 9$ & $\mathrm{X} 10$ & $\mathrm{X} 11$ \\
\hline $\begin{array}{c}\text { Value } \\
{[\mathrm{mm}]}\end{array}$ & 10 & 10 & 12 & 14 & 12 & 12 & 12 & 18 & 12 & 12 & 10 \\
\hline
\end{tabular}

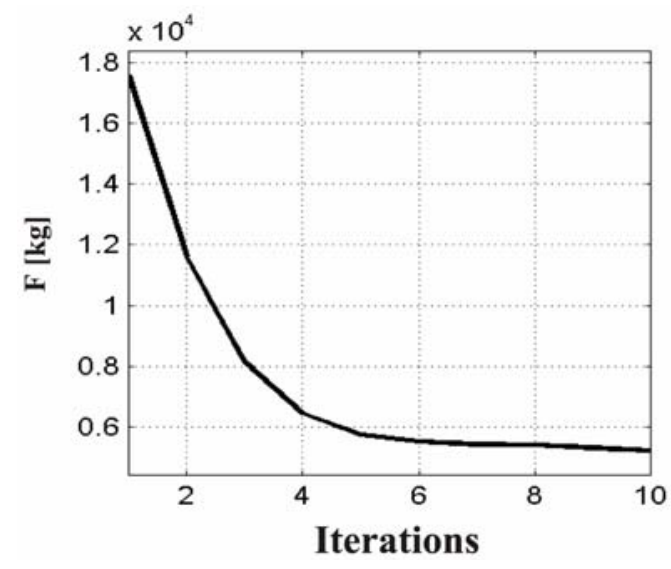

Fig. 9 Reduction of the structural weight

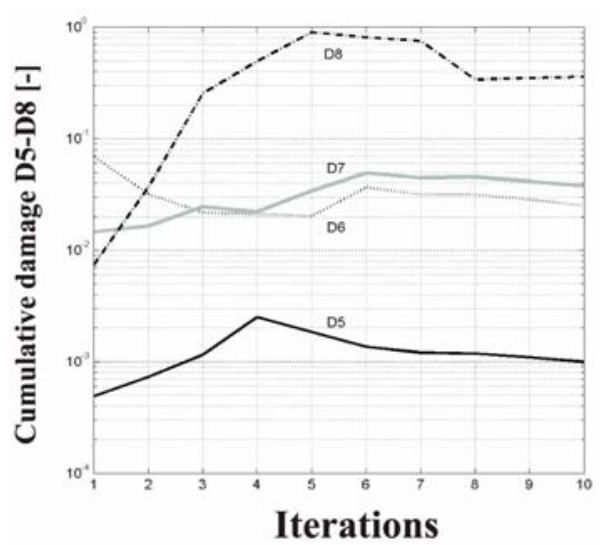

Fig. 10 Convergence history of the cumulative damage for optimizing groups $X 5-X 8$

\section{Conclusion}

The goal of the paper was to present some multi-axial fatigue damage computational approaches and implementation and application of the chosen structural optimization technique in the case of the thin shell vehicle frames. The used methods were in-built into finite element analysis. The results of numerical studies verify accuracy of the applied optimizing approach in structural designing 
with fatigue damage conditions. It is also necessary to remember a general problem of computational mechanics, i.e. time-consuming calculation hence in the authors' opinion the proposed algorithms will be acceptable in technical community.

\section{Acknowledgements}

This work has been supported by VEGA grant No. 1/0125/09 and $1 / 0727 / 10$

\section{References}

[1] BATHE, K. J.: Finite Element Procedures. New Persey : Prentice Hall, 1996.

[2] CARPINTERI, A., SPAGNOLI, A., VANTADORI, S.: A Multiaxial Fatigue Criterion for Random Loading, Special Issue of Fatigue and Fracture of Engineering Materials and Structures, Vol.26, 6/2003, pp. 515-522.

[3] HAFTKA, R. T., GURDAL, Z., KAMAT, M.: Elements of Structural Optimization. Kluwer, Dordrecht, 1990.

[4] KWON, Y. W., BANG, H.: The Finite Element Method using MATLAB. CRC Press : University of Minnesota, 1996.

[5] PITOISET, X., PREUMONT, A., KERNILIS, A.: Tools for a Multiaxial Fatigue Analysis of Structures Submitted to Random Vibrations. Proc. of European Conference on Spacecraft Structures Materials and Mechanical Testing, Braunschweig, Germany, 1998.

[6] PREUMONT, A., PIEFORT, V.: Predicting Random High Cycle Fatigue Life with Finite Elements. Journal of Vibration and Acoustics, 116, 1994, pp. 245-248.

[7] SAGA, M., MEDVECKY, S., KOPECKY, M.: The Effective Algorithm for Discrete Structural Mass Minimising Subjected to Fatigue Life. 6th World Congress on Structural and Multidisciplinary Optimization, Rio de Janeiro, Brasil, May 2005, (full paper on CD).

[8] SAGA, M., VASKO M.: Stress Sensitivity Analysis of the Beam and Shell Finite Elements. Communications - Scientific Letters of the University Zilina, Vol. 11, No. 2, 2009, pp. 5-12.

[9] SAGA, M., VASKO, M., JANDACKA, J., HOLKOVA, Z.: Contribution to Stress Sensitivity Analysis of the Shell Finite Elements. Applied and Computational Mechanics, Vol. II, No. 1, 2008, pp. 113-122.

[10] SAGA, M., VAVRO, J.: Contribution to Non-Proportional Multiaxial Fatigue Analysis by FEM. Materials Engineering, Vol. 11, No. 1, 2004, pp. 143-150.

[11] SAGA, M.: Mass Minimising of Truss Structures Subjected to Prescribed Fatigue Life. Machine Dynamics Problems, Vol. 28, No. 4, 2004, pp. 101-106.

[12] SAGA, M.: Optimising Techniques for Multiaxial Fatigue Analysis by FEM. Computational Mechanics, 2002, Nectiny, 2002, pp. 403-408. 